\title{
Searching for Dark Particles with Quantum Optics
}

\author{
J. Estrada,,${ }^{1, *}$ R. Harnik $\odot,{ }^{1, \dagger}$ D. Rodrigues,,${ }^{1,2, \ddagger}$ and M. Senger $\circledast^{2,3, \S}$ \\ ${ }^{1}$ Fermi National Accelerator Laboratory, P.O. Box 500, Batavia, Illinois 60510, USA \\ ${ }^{2}$ Department of Physics, FCEN, University of Buenos Aires and IFIBA, CONICET, Buenos Aires, Argentina \\ ${ }^{3}$ Physik-Institut der Universität Zürich, 8057 Zürich, Switzerland
}

(Received 19 January 2021; revised 13 May 2021; accepted 28 July 2021; published 7 September 2021)

\begin{abstract}
We propose a way to use optical tools from quantum imaging and quantum communication to search for physics beyond the standard model. Spontaneous parametric down-conversion (SPDC) is a commonly used source of entangled photons in which pump photons convert to a signal-idler pair. We propose to search for "dark-SPDC" (dSPDC) events in which a new dark-sector particle replaces the idler. Though it does not interact, the presence of a dark particle can be inferred by the properties of the signal photon. Examples of dark states include axionlike particles and dark photons. We show that the presence of an optical medium opens the phase space of the down-conversion process, or decay, which would be forbidden in a vacuum. Search schemes are proposed that employ optical imaging and/or spectroscopy of the signal photons. The signal rates in our proposal scales with the second power of the small coupling to new physics, as opposed to light-shining-through-wall experiments, the signal of which scales with coupling to the fourth power. We analyze the characteristics of the optical media needed to enhance dSPDC and estimate the rate.
\end{abstract}

DOI: 10.1103/PRXQuantum.2.030340

\section{INTRODUCTION}

Nonlinear optics is a powerful new tool for quantum information science. Among its many uses, it plays an enabling role in the areas of quantum networks and teleportation of quantum states, as well as in quantum imaging. In quantum teleportation [1-4], the state of a distant quantum system can be inferred without direct interaction but, rather, by allowing it to interact with one of the photons in an entangled pair. The coherence of these optical systems has recently allowed teleportation over a large distance [5]. Quantum ghost imaging, or "interaction-free" imaging [6], is used to discern (usually classical) information about an object without direct interaction. This technique exploits the relationship among the emission angles of a correlated photon pair to create an image with high angular resolution without placing the subject in front of a high-resolution detector or allowing it to interact with intense light. These

\footnotetext{
*estrada@fnal.gov

†roni@fnal.gov

†rodriguesfm@df.uba.ar

§matias.senger@physik.uzh.ch
}

Published by the American Physical Society under the terms of the Creative Commons Attribution 4.0 International license. Further distribution of this work must maintain attribution to the author(s) and the published article's title, journal citation, and DOI. methods of teleportation and imaging rely on the production of signal photons in association with an idler pair that is entangled (or at least correlated) in its direction, frequency, and sometimes polarization.

Quantum ghost imaging and teleportation both differ parametrically from standard forms of information transfer. This is because a system is probed not by sending information to it and receiving information back but, rather, by sending it half of an EPR pair, without the need for a "response." The difference is particularly apparent if the subject is an extremely weakly coupled system - say, it is part of a dark sector - with a coupling $\epsilon$ to photons. The information flow in capturing an image will occur at a rate $\propto \epsilon^{4}$ with standard methods but at a rate $\propto \epsilon^{2}$ using quantum optical methods.

The parametric scaling of rates with $\epsilon^{4}$ for conventional imaging is similar to many searches for new particles that are light and weakly coupled, dark photons and axions (which is introduced below), where the new states are emitted and detected in the laboratory. Both emission and detection scale with the square of the coupling, leading to an overall rate of $\epsilon^{4}$. The goal of this work is to adopt an "interaction-free" method to search for these new particles, inspired by quantum teleportation and ghost imaging and using some of their tools, to achieve an $\epsilon^{2}$ scaling of signal rates. This can be done if the new particle is produced in association with a signal photon that is used for detection, as illustrated in Fig. 1. 


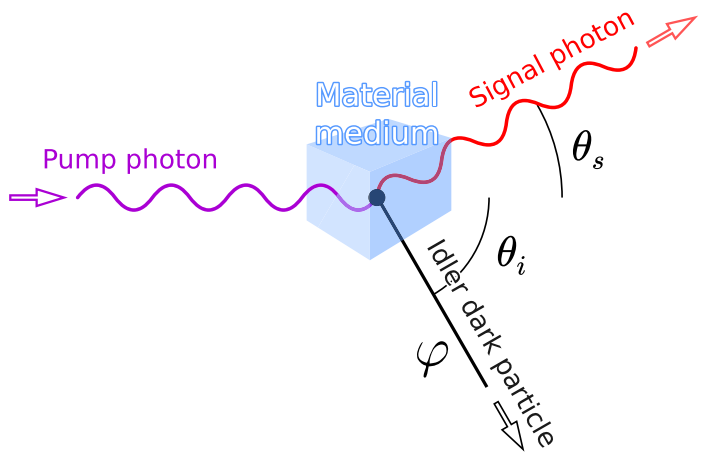

FIG. 1. A pictorial representation of the dSPDC process. A dark particle $\varphi$ is emitted in association with a signal photon. The presence of $\varphi$ can be inferred from the distribution of the signal photon in angle and/or frequency. We consider both the collinear $\left(\theta_{s}=0\right)$ and noncollinear $\left(\theta_{s} \neq 0\right)$ cases.

A common method for generating entangled photon pairs in teleportation and imaging is the nonlinearoptics process known as spontaneous parametric downconversion (SPDC). In SPDC, a pump photon decays, or down-converts, within a nonlinear optical medium into two other photons, a signal and an idler. The presence of the SPDC idler can be inferred by the detection of the signal [7]. SPDC is in wide use in quantum information and provides the seed for cavity-enhanced setups such as optical parametric oscillators (OPOs) [8].

In this work, we propose to use quantum imaging and quantum communication tools to perform an interactionfree search for the emission of new particles beyond the standard model. The tool that we present is dark SPDC, or dSPDC, an example sketch of which is shown in Fig. 1. A pump photon enters an optical medium and down-converts to a signal photon and a dark particle, which can have a small mass, and does not interact with the optical medium. As in SPDC, in dSPDC the presence of a dark state can be inferred by the angle and frequency distribution of the signal photon that is produced in association. The dSPDC process can occur either collinearly, with $\theta_{s}=\theta_{i}=0$, or as shown in the sketch, in a noncollinear way.

The new dark particles that we propose to search for, low in mass and very weakly interacting, are simple extensions of the standard model (SM). Among the most well motivated are axions, or axionlike particles, and dark photons (for a review, see Ref. [9]). Since these particles interact with SM photons, optical experiments provide an opportunity to search for them. A canonical setup is the light-shining-through-wall (LSW) experiment, which sets interesting limits on axions and dark photons using optical cavities [10]. In optical LSW experiments, a laser photon converts to an axion or dark photon, enabling it to penetrate an opaque barrier. For detection, however, the dark state must convert back to a photon and interact, which is a rare occurrence. As a result, the signal rate in LSW scales as $\epsilon^{4}$, where $\epsilon$ is a small coupling to dark states. This motivates the interaction-free approach of dSPDC, in which the dark state is produced but does not interact again, yielding a rate $\propto \epsilon^{2}$.

From the perspective of particle physics, dSPDC, and also SPDC, may sound unusual [11]. In dSPDC, for example, a massless photon is decaying to another massless photon plus a massive particle. In the language of particle physics, this would be a kinematically forbidden transition. If it were to happen in a vacuum, the process would violate energy and momentum conservation and thus have no available phase space. In this paper, we show how optics enables us to perform "engineering in phase space" and open up kinematics to otherwise forbidden channels. This, in turn, allows us to design setups in which the dSPDC process is allowed and can be used to search for dark particles.

Broadly speaking, the search strategies we propose may be classified as employing either imaging or spectroscopic tools, though methods employing both of these are also possible. In the first, the angular distribution of signal photons are measured, while in the second, the energy distribution is observed. For an imaging-based search, a high angular resolution is required, while the spectroscopic approach requires high frequency resolution. The latter has the benefit that dSPDC can be implemented in a waveguide, which will enhance its rate for long optical elements. This is well established [12] for SPDC and will be derived in detail in Ref. [13] for dSPDC. We note that, recently, SPDC has been proposed as a tool to search for the conversion of axionlike particles to photons in a magnetic field, exploiting the reduced variance in photon number in a setup with a movable mirror [14]. This proposal, though its rate also scales with the square of the axion coupling and also makes use of nonlinear optical tools, is qualitatively different than our dSPDC proposal, e.g., in the phase-matching conditions and in the presence of a magnetic field.

We present the basic ideas and formalism behind this method and focus on the phase space for dSPDC. We compare the phase-space distributions of SPDC to dSPDC and discuss factors that may enhance the rate of dSPDC processes. To estimate the dSPDC rates, in this work we rescale known SPDC results. In a forthcoming paper [13], we will derive the dSPDC rate more carefully, focusing on the spectroscopic approach and on collinear dSPDC in bulk crystals and waveguides.

This paper is structured as follows. In Sec. II, we review axionlike particles and dark photons and present their Hamiltonians in the manner in which they are usually treated in nonlinear optics. In Sec. III, we discuss energy and momentum conservation, which are called phase-matching conditions in nonlinear optics, and the phase space for dSPDC. The usual conservation rules will hold exactly in a thought experiment of an infinite optical medium. In Sec. III C, we discuss how the finite size of 
the optical medium broadens the phase-space distribution and plot it as a function of the signal-photon angle and energy. In Sec. IV, we discuss the choice of optical materials that allow for the dSPDC phase matching to enhance its rates and to suppress or eliminate SPDC backgrounds. In Sec. V, we discuss the rates for dSPDC and its scaling with the geometry of the experiment, showing potential promising results for collinear waveguide setups with long optical elements. We discuss future directions and conclude in Sec. VI.

\section{THE DARK-SPDC HAMILTONIAN}

The SPDC process can be derived from an effective nonlinear optics Hamiltonian of the form $[15,16]$

$$
\mathcal{H}_{\text {SPDC }}=\chi^{(2)} E_{i} E_{s} E_{p},
$$

where $E_{j}$ is the electric field for the pump, signal, and idler photons and $j=p, s, i$, respectively. . We adopt the standard notation in nonlinear optics literature in which the vector nature of the field is implicit. Thus the pump, signal, and idler can each be of a particular polarization and $\chi^{(2)}$ represents the coupling between the corresponding choices of polarization. The Hamiltonian of Eq. (1) is an effective field theory defined at length scales of the order of the wavelength of optical light (or longer). At the microscopic level, optics and nonlinear optics arise from the collective back reaction of dipoles in the medium on light that is traversing it (see, e.g., Ref. [17]. At the linear level, this leads to indices of refraction that differ from unity. Nonlinear phenomena arise due to the anharmonic material effect. The fields in Eq. (1) can be expanded in modes and their creation or annihilation operators to describe SPDC (see, e.g., Refs. $[12,15,16,18]$.

We now consider the dark-SPDC Hamiltonian

$$
\mathcal{H}_{\mathrm{dSPDC}}=g_{\varphi} \varphi E_{S} E_{p},
$$

in which a pump and signal photon couple to a new field $\varphi$ that has a mass $m_{\varphi}$. The effective coupling, $g_{\varphi}$, depends on the model and can depend on the frequencies in the process, the polarizations, and the directions of outgoing particles. Like the SPDC case, this Hamiltonian is an effective theory at or below optical energy scales, though the coupling strength may be set at much higher scales, as we will see below. Indeed, this type of term arises in wellmotivated dark-sector models including axionlike particles [19-21] and dark photons [22] (for a review, see also Ref. [9]).

\section{A. Axionlike particles}

Axionlike particles, also known as ALPs, are light pseudoscalar particles that couple to photons via a term

$$
\mathcal{H}_{\text {axion }}=g_{\varphi} \varphi \vec{E} \cdot \vec{B}
$$

They are naturally light due to a spontaneously broken global symmetry at a scale $f_{a}$. The coupling in Eq. (3) is typically generated at a very high energy scale and is a valid effective theory at low energies. When this interaction is describing interactions of optical photons, the photon degrees of freedom in the interaction will have indices of refraction that differ from unity. This opens the phase space for the dSPDC process. When $\vec{E}$ and $\vec{B}$ are expanded as quantum fields, they will create or annihilate photons of different polarizations in the collinear configuration. The axion Hamiltonian can then be written in the scalar form of Eq. (2), with the pump and signal photons chosen to have orthogonal polarization. The coupling $g_{\varphi}$ in this case is the usual axion-photon coupling of approximately $1 / f_{a}$ (with a frequency-dependent coefficient of order $k / \omega)$.

Since we do not perform a detailed analysis of the backgrounds and feasibility here, we describe the existing limits on ALPs qualitatively and refer readers to limit plots in Refs. [9,23]. In this work, we consider searches that can probe ALPs with a mass of order $0.1 \mathrm{eV}$ or lower. The existing limits in this mass range come from stellar and supernova dynamics [24], as well as from the CAST Helioscope [25], and require $g_{\varphi} \lesssim 10^{-10} \mathrm{GeV}^{-1}$. The planned helioscopes BabyIAXO [26] and IAXO [27] will further improve on the reach of CAST. For comparison with our setups, we also consider limits that are entirely laboratory based, which are much weaker. At approximately $0.1 \mathrm{eV}$, an ALP coupling of order $10^{-6} \mathrm{GeV}^{-1}$ is allowed by all laboratory-based searches. For axion masses around $10^{-3}-10^{-2} \mathrm{eV}$, the "Polarisation of Vacuum with LASer" (PVLAS) search[28] for magnetic birefringence places a limit of $g_{\varphi} \lesssim 10^{-7}-10^{-6} \mathrm{GeV}^{-1}$. Below masses of an millielectronvolt, LSW experiments such as the "Optical Search for QED Vacuum Bifringence, Axions and Photon Regeneration" (OSQAR) [29] and the "Any Light Particle Search" (ALPS) [30] set $g_{\varphi} \lesssim 6 \times 10^{-8}$. A largescale LSW facility, ALPS II [31], is proposed in order to improve upon the astrophysical limits and CAST.

It is interesting to note that unlike LSW axion searches, dSPDC does not require an external magnetic field to be present, even though both processes use Eq. (3). Because the Hamiltonian involves three fields and the $1 \rightarrow 1$ photon-to-axion conversion only involve two fields, a magnetic field in the background is required. By contrast, the dSPDC is a $1 \rightarrow 2$ process and all three fields are contracted with the initial or final states.

\section{B. Dark photons}

The dark photon [22] is a new $\mathrm{U}(1)$ gauge field, $A^{\prime}$, with mass $m_{A^{\prime}}$, that mixes kinetically with the photon via the Hamiltonian

$$
\mathcal{H}_{\text {mix }}=\epsilon F^{\mu \nu} F_{\mu \nu}^{\prime}=\epsilon\left(\vec{E} \cdot \vec{E}^{\prime}+\vec{B} \cdot \vec{B}^{\prime}\right) .
$$


It is possible to write the dark-photon interaction in a basis in which any electromagnetic current couples to longitudinally polarized dark photons with an effective coupling of $\epsilon\left(m_{A^{\prime}} / \omega_{A^{\prime}}\right)$ and to transversely polarized dark photons with $\epsilon\left(m_{A^{\prime}} / \omega_{A^{\prime}}\right)^{2}[32,33]$. The same dynamics that lead to the nonlinear Hamiltonian $\mathcal{H}_{\text {SPDC }}$ will yield an effective coupling of two photons to a dark photon as in Eq. (2), with $\varphi$ representing a dark-photon polarization state. For longitudinal polarization of the dark photon, this can be brought into the form of Eq. (2), with $g_{\varphi}=\epsilon \chi^{(2)} m_{\varphi}$. The coupling to transverse dark photons will be suppressed by an additional factor of $m_{\varphi} / \omega_{\varphi}$.

Like axions, limits on dark photons come from astrophysical systems, such as energy loss in the Sun [32], as well as the lack of a detection of dark photons emitted by the Sun in dark-matter direct-detection experiments [33]. At a dark-photon mass of order $0.1 \mathrm{eV}$, the limit on the kinetic mixing is $\epsilon \lesssim 10^{-10}$, with the limit becoming weaker linearly as the dark-photon mass decreases. Among purely laboratory-based experiments, ALPS sets a limit of $\epsilon \lesssim 3 \times 10^{-7}$. In forthcoming sections of this work, we use these limits as benchmarks.

In addition to ALPs and dark photons, one can consider other new dark particles that are sufficiently light and that couple to photons. Examples include millicharged particles [22] (also reviewed in Ref. [9]), which can be produced in pairs in association with a signal photon. We leave these generalizations for future work and proceed to discuss the phase space for dSPDC in a model-independent way.

\section{PHASE SPACE OF DARK SPDC}

As stated above, the effective energy and momentum conservation rules in nonlinear optics are known as "phasematching conditions." We begin by reviewing their origin to make the connection with energy and momentum conservation in particle physics and then proceed to solve them for SPDC and dSPDC, to understand the phase space for these processes.

\section{A. Phase matching and particle decay}

We begin with a discussion of the phase space in a twobody decay, or spontaneous down-conversion. We use the language of particle physics, but we label the particles as is usual in nonlinear optics systems, which we discuss from the onset. A pump particle $p$ decays into a signal particle $s$ and another particle, either an idler photon $i$ or a dark particle $\varphi$ :

$$
\begin{aligned}
\text { SPDC: } & \gamma_{p} \rightarrow \gamma_{s}+\gamma_{i} \\
\mathrm{dSPDC}: & \gamma_{p} \rightarrow \gamma_{s}+\varphi .
\end{aligned}
$$

We discuss the kinematics of the standard-model SPDC process and the beyond-the-SM (BSM) dSPDC process together, to compare and contrast. For this, in the discussion below we use the idler label $i$ to represent the idler photon in the SPDC case and $\varphi$ in the dSPDC case. Hence $\mathbf{k}_{i}$ and $\theta_{i}$ are the momentum and emission angle of either the idler photon or of $\varphi$, depending on the process.

The differential decay rate in the laboratory frame is given by [23]

$$
\frac{d \Gamma}{d^{3} k_{s} d^{3} k_{i}}=\frac{\delta^{4}\left(p_{p \mu}-p_{s \mu}-p_{i \mu}\right)}{(2 \pi)^{2} 2 \omega_{p} 2 \omega_{s} 2 \omega_{i}}|\mathcal{M}|^{2},
$$

where $\mathcal{M}$ is the amplitude for the process (which carries a mass dimension of +1 ) and the energy-momentum four vectors

$$
p_{j \mu}=\left(\begin{array}{c}
\omega_{j} \\
\mathbf{k}_{j}
\end{array}\right)
$$

with $j=p, s, i$. Traditionally, one proceeds by integrating over 4 degrees of freedom within the six-dimensional phase space, leaving a differential rate with respect to the two-dimensional phase space. This is often chosen as a solid angle for the decay $d \Omega$ but in our case there will be nontrivial correlations of the frequency and the angle, as we will see in the next subsection.

It is instructive, however, for our purpose to take a step back and recall how the energy-momentum-conserving delta functions come about. In calculating the quantum amplitude for the transition, the fields in the initial and final states are expanded in modes. The plane-wave phases $e^{i(\omega t-\mathbf{k} \cdot \mathbf{x})}$ are collected from each and a space-time integral is performed:

$$
\int d^{4} x e^{i(\Delta \omega t-\Delta \mathbf{k} \cdot \mathbf{x})}=(2 \pi)^{4} \delta^{(3)}(\Delta \mathbf{k}) \delta(\Delta \omega),
$$

where the frequency and momentum mismatch are defined as

$$
\left\{\begin{array}{l}
\Delta \omega=\omega_{p}-\omega_{s}-\omega_{i}, \\
\Delta \mathbf{k}=\mathbf{k}_{p}-\mathbf{k}_{s}-\mathbf{k}_{i},
\end{array}\right.
$$

and, again, the label $i$ can describe either an idler photon or a dark particle $\varphi$. In Eq. (9), we separate the momentumand energy-conserving delta functions to pay homage to the optical systems that will be the subject of upcoming discussion. The squared amplitude $|\mathcal{M}|^{2}$, and hence the rate, is proportional to a single power of the energymomentum-conserving delta function times a space-time volume factor, which is absorbed for canonically normalized states (see, e.g., Ref. [34]), giving Eq. (6).

The message from this is that energy and momentum conservation, which are a consequence of Neother's theorem and space-time translation symmetry, is enforced in quantum field theory by perfect destructive interference 
whenever there is a nonzero mismatch in the momentum or energy of initial and final states. In this sense, the term "phase matching" captures the particle physicist's notion of energy and momentum conservation well.

The dSPDC process shown in Fig. 1 is a massless pump photon decaying to a signal photon plus a massive particle $\varphi$. This process clearly cannot occur in a vacuum. For example, if we go to the rest frame of $\varphi$ and define all quantities in this frame with a tilde $\tilde{\tilde{\mathbf{k}}}$, conservation of momentum implies that $\tilde{\mathbf{k}}_{p}=\tilde{\mathbf{k}}_{s}$, while conservation of energy implies that $\tilde{\omega}_{p}=\tilde{\omega}_{s}+m$. Combining these with the dispersion relation for a photon in a vacuum, $\tilde{\omega}_{p, s}=$ $\left|\tilde{\mathbf{k}}_{p, s}\right|$, this implies that energy and momentum conservation cannot be satisfied and that the process is kinematically forbidden. Another way to view this is to recall that the energy-momentum four vector for the initial state (a photon) lies on a light cone, i.e., it is a null four vector, $p^{\mu} p_{\mu}=0$, while the final state cannot accomplish this, since there is a nonzero mass.

In this work, we show that optical systems will allow us to open phase space for dSPDC. When a photon is inside an optical medium, a different dispersion relation holds,

$$
\tilde{n}_{p} \tilde{\omega}_{p}=\left|\tilde{\mathbf{k}}_{p}\right| \quad \text { and } \quad \tilde{n}_{s} \tilde{\omega}_{s}=\left|\tilde{\mathbf{k}}_{s}\right|,
$$

where $\tilde{n}_{p}$ and $\tilde{n}_{s}$ are indices of refraction of photons in the medium in the $\varphi$ rest frame, which can be different for the pump and the signal. As we will see later on, under these conditions the conclusion that $p \rightarrow s+\varphi$ is kinematically forbidden can be evaded.

Another effect that occurs in optical systems, but not in decays in particle physics, is the breaking of spatialtranslation invariance by the finite extent of the optical medium. This allows for violation of momentum conservation along the directions in which the medium is finite. As a result, the sharp momentum-conserving delta function will become a peaked distribution of width $L^{-1}$, where $L$ is the crystal size. We begin by solving the exact phase-matching conditions in SPDC and dSPDC, which correspond to the phase-space distributions for an infinitely large crystal, in the following subsection. Next, we move on to the case in which the optical medium is finite.

\section{B. Phase matching in dSPDC}

We now study the phase space for dSPDC to identify the correlations between the emission angle and the frequency of the signal photon. We also consider the SPDC process in parallel, so that in the end we arrive at both results. In this subsection, we assume an optical medium with an infinite extent, such that the delta functions enforce energy and momentum conservation.

The phase-matching conditions $\Delta \omega=0$ and $\Delta \mathbf{k}=$ 0 - which must be strictly satisfied in the infinite-extent optical medium scenario-imply that

$$
\left\{\begin{array}{l}
\omega_{p}=\omega_{s}+\omega_{i} \\
\mathbf{k}_{p}=\mathbf{k}_{s}+\mathbf{k}_{i}
\end{array} .\right.
$$

Using the decomposition of $\mathbf{k}$ shown in Fig. 1, the second equation can be expanded in coordinates parallel and perpendicular to the pump propagation direction:

$$
\left\{\begin{array}{l}
k_{p}=k_{s} \cos \theta_{s}+k_{i} \cos \theta_{i}, \\
0=k_{s} \sin \theta_{s}-k_{i} \sin \theta_{i},
\end{array}\right.
$$

where the angles $\theta_{s}$ and $\theta_{i}$ are those indicated in the cited figure. Since this process is happening in a material medium, the photon dispersion relation is $k=n \omega$, where $n$ is the refractive index. The refractive index is, in general, a function of the frequency, polarization, and direction of propagation, and can thus be different for pump, signal, and idler photons. For dSPDC, in which the $\varphi$ particle is massive and weakly interacting with matter, the dispersion relation is the same as in a vacuum:

$$
k_{\varphi}=\sqrt{\omega_{\varphi}^{2}-m_{\varphi}^{2}} .
$$

Thus we can explicitly write the dispersion relation for each particle in this process:

$$
\begin{aligned}
k_{p} & =n_{p} \omega_{p}, \\
k_{s} & =n_{s} \omega_{s},
\end{aligned}
$$

and

$$
k_{i}=\left\{\begin{array}{ll}
n_{i} \omega_{i}, & \text { for SPDC, } \\
\sqrt{\omega_{i}^{2}-m_{\varphi}^{2}}, & \text { for dSPDC }
\end{array} .\right.
$$

where, again, the label " $i$ " refers to an idler photon for SPDC and to the dark field $\varphi$ for dSPDC. Note that $k_{i}$ for SPDC not only has $m=0$ but also takes into account the "strong (electromagnetic) coupling" with matter summarized by the refractive index $n_{i}$. As a result, for SPDC we always have $k_{i}>\omega_{i}$, while for dSPDC $k_{i}<\omega_{i}$. Substituting these dispersion relations into Eq. (12) and using the first equation in Eq. (11), we obtain the phase-matching relation of the signal angle and the frequency:

$$
\cos \theta_{s}=\frac{n_{p}^{2}+\alpha_{\omega}^{2} n_{s}^{2}-\Xi^{2}}{2 \alpha_{\omega} n_{p} n_{s}}
$$

where we define

$$
\alpha_{\omega} \stackrel{\text { def }}{=} \frac{\omega_{s}}{\omega_{p}}
$$


and

$$
\Xi \stackrel{\text { def }}{=} \frac{k_{i}}{\omega_{p}}= \begin{cases}n_{i}\left(1-\alpha_{\omega}\right), & \text { for SPDC, } \\ \sqrt{\left(1-\alpha_{\omega}\right)^{2}-\frac{m_{\varphi}^{2}}{\omega_{p}^{2}},} & \text { for dSPDC }\end{cases}
$$

Equation (17) defines the phase space for (d)SPDC, along with the azimuthal angle $\phi_{s}$. The idler angle, or that of the dark particle in the dSPDC case, is fixed in terms of the signal angle and frequency by requiring conservation of transverse momentum:

$$
\begin{aligned}
\sin \theta_{i} & =\frac{k_{s}}{k_{i}} \sin \theta_{s}, \\
\phi_{i} & =\phi_{s}+\pi,
\end{aligned}
$$

where $k_{i}$ is evaluated at a frequency of $\omega_{i}=\omega_{p}-\omega_{s}$ and $k_{s}$ at the frequency $\omega_{s}$ according to the dispersion relation in Eqs. (15) and (16).

In the left panel of Fig. 2, we show the allowed phase space in the $\alpha_{\omega}-\theta_{s}$ plane for SPDC and for dSPDC with both a massless and a massive $\varphi$. Here, we choose $n_{p}=$ 1.486 and $n_{s}=1.658$, with $n_{i}=n_{s}$ in the SPDC case. These values are chosen to be constant with the frequency and propagation direction, for simplicity, and are inspired by the ordinary and extraordinary refractive indices in calcite and are used as a benchmark in some of the examples below.

We see that phase matching is achieved in different regions of phase space for SPDC and its dark counterpart.

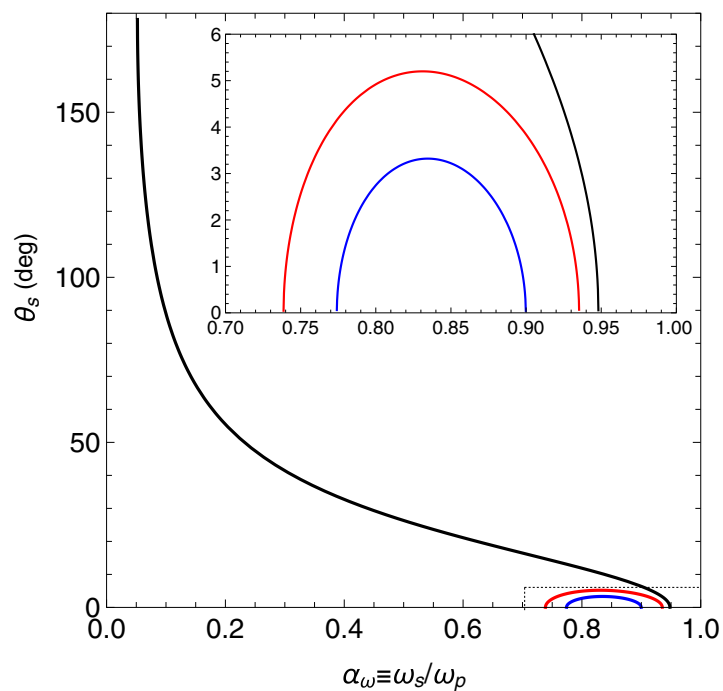

In dSPDC, signal emission angles are restricted to near the forward region and in a limited range of signal frequencies. The need for more forward emission in ASPDC can be understood because $\varphi$ effectively sees an index of refraction of one. This implies that it can carry less momentum for a given frequency, as compared to a photon that obeys $k=n \omega$. As a result, the signal photon must point nearly parallel to the pump, in order to conserve momentum (see Fig. 2, right). An additional difference to notice is that, in contrast with the SPDC example shown, for a fixed signal emission angle there are two different signal frequencies that satisfy the phase matching in dSPDC. This will always be the case for dSPDC. Although SPDC can be of this form as well, typical refractive indices usually favor single solutions, as shown for calcite.

\section{Phase matching for collinear dSPDC}

One case of particular interest is that of collinear dSPDC, in which the emission angle is zero, as would occur in a single-mode fiber or a waveguide. For this case, so long as $n_{s}>n_{p}$, an axion mass below some threshold can be probed. Setting the emission angles to zero, there are two solutions to the phase-matching equations, which give signal-photon energies of

$$
\alpha_{\omega}=\frac{\left(n_{s} n_{p}-1\right) \pm \sqrt{\left(n_{s}-n_{p}\right)^{2}-\left(n_{s}^{2}-1\right) \frac{m_{\varphi}^{2}}{\omega_{p}^{2}}}}{n_{s}^{2}-1} .
$$

SPDC

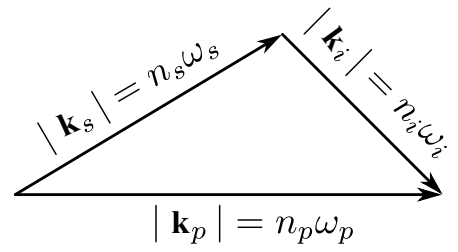

dSPDC

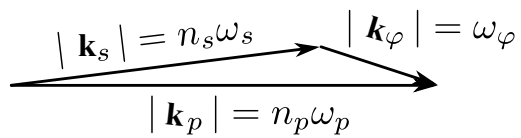

FIG. 2. Left: the allowed phase space for SPDC (black), dSPDC with $m=0$ (red), and dSPDC with $m=0.1 \omega_{p}$ (blue), shown as in the plane of signal emission angle as a function of frequency ratio $\alpha_{\omega}$. The indices of refraction here are $n_{p}=1.658$ and $n_{s}=1.486$ as an example, inspired by calcite. The inset shows an enlargement of the dSPDC phase space. Right: sketches depicting the momentum phase-matching condition $\Delta \mathbf{k}=0$ for SPDC and dSPDC, with massless $\varphi$. In both cases, we take the same $\omega_{p}$ and $\omega_{s}$. Due to the index of refraction for $\varphi$ being essentially one and that for the idler photon being larger (say, approximately 1.5), phase matching in dSPDC has a smaller signal emission angle than that of SPDC. 


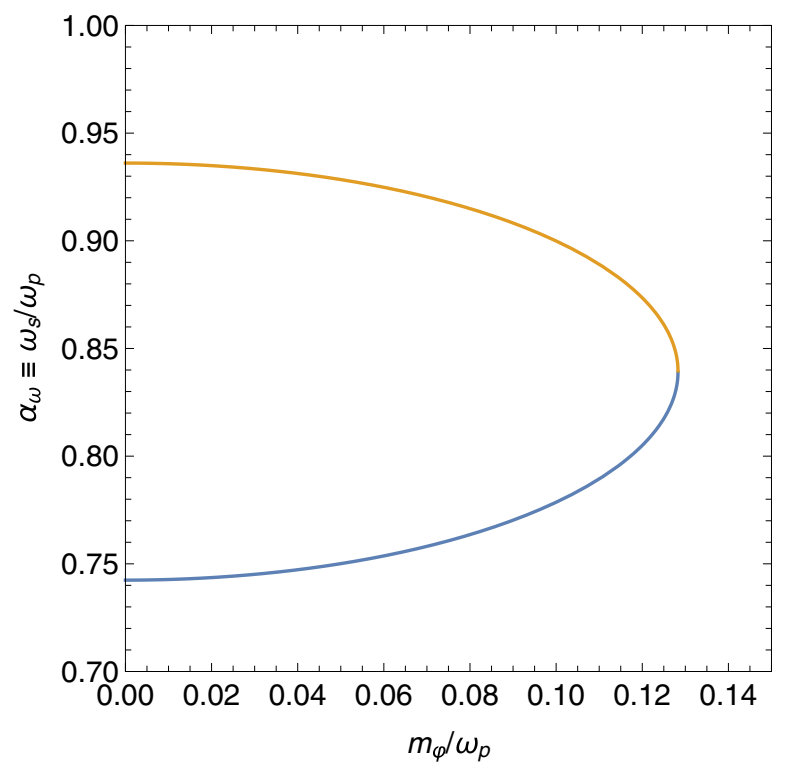

FIG. 3. Solutions to the phase-matching conditions for the collinear dark-SPDC process for the signal-photon energy as a function of the mass of a dark particle $\varphi$. This phase space is relevant for waveguide-based experiments. Both axes are normalized to the pump frequency. The two branches correspond to configurations in which $\varphi$ is emitted forward (bottom) and backward (top).

The + solution above corresponds to the case where the $\varphi$ particle is emitted in the forward direction, whereas the represents the case where it is emitted backward. The signal frequency as a function of the $\varphi$ mass is shown in Fig. 3 for the calcite benchmark. For the near-massless case, $m_{\varphi} \ll \omega_{p}$, a phase-matching solution exists for $n_{s} \geq n_{p}$, giving

$$
\frac{\omega_{s}}{\omega_{p}}=\frac{n_{p} \mp 1}{n_{s} \mp 1} .
$$

For calcite, we obtain $\omega_{s}=0.739 \omega_{p}$ and $\omega_{s}=0.935 \omega_{p}$ for forward- and backward-emitted axions, respectively.

Of course, not just any combination of $n_{p}$ and $n_{s}$ allows us to achieve phase matching in dSPDC. We can state here that $n_{s}>n_{p}$ is a requirement for phase matching to be possible for a massless $\varphi$ and that $n_{s}-n_{p}$ must grow as $m_{\varphi}$ grows. We discuss these and other requirements for dSPDC searches in Sec. IV.

\section{Thin planar layer of optical medium}

We now consider the effects of a finite crystal. Specifically, we assume that the optical medium is a planar thin layer [35] of optical material of length $L$ along the pump propagation direction $\hat{z}$ and that it is infinite in transverse directions. In this case, the integral in Eq. (8) is performed only over a finite range and the delta function is replaced by a sinc $x \equiv \sin x / x$ function

$$
\int_{-L / 2}^{L / 2} d z e^{i \Delta k_{z} z}=L \operatorname{sinc}\left(\frac{\Delta k_{z} L}{2}\right)
$$

at the level of the amplitude. As expected, this allows for momentum nonconservation with a characteristic width of order $L^{-1}$.

The fully differential rate will be proportional to the square of the sinc function

$$
\begin{aligned}
\frac{d \Gamma}{d^{3} k_{p} d^{3} k_{i}} & \propto L^{2} \operatorname{sinc}^{2}\left(\frac{\Delta k_{z} L}{2}\right) \delta^{2}\left(\Delta \mathbf{k}_{T}\right) \delta(\Delta \omega) \\
& \equiv \frac{d \hat{\Gamma}}{d^{3} k_{p} d^{3} k_{i}}
\end{aligned}
$$

where $\Delta \mathbf{k}_{T}$ is the momentum-mismatch vector in the transverse directions. The constant of proportionality in Eq. (24) will have frequency-normalization factors of the form $(2 \omega)^{-1}$ and the matrix element $\mathcal{M}$. The matrix element can also have a nontrivial angular dependence on $\theta$ as well as on $\phi$, depending on the model and the optical medium properties. Note, however, that the current analysis for the phase space is model independent. We thus postpone the discussion of overall rates to Sec. V (and in greater detail in forthcoming work [13]) and here we limit the study to the phase-space distribution only. We proceed with the defined phase-space distribution $d \hat{\Gamma}$ in Eq. (25), which we now study.

One can trivially perform the integral over the $d^{3} k_{i}$, which will effectively enforce Eq. (20) for transverse momentum conservation, and set $\left|k_{i}\right|$ by conservation of energy. The argument of the sinc function is

$$
\begin{aligned}
\Delta k_{z} & =k_{p}-k_{s} \cos \theta_{s}-k_{i} \cos \theta_{i} \\
& =\omega_{p}\left(n_{p}-n_{s} \alpha_{\omega} \cos \theta_{s} \pm \sqrt{\Xi^{2}-n_{s}^{2} \alpha_{\omega}^{2} \sin ^{2} \theta_{s}},\right)
\end{aligned}
$$

where in the second step we use Eq. (20) and the definitions of $\alpha_{\omega}$ and $\Xi$ in Eqs. (18) and (19). The \pm sign accounts for the idler or dark particle being emitted in the forward and backward direction, respectively.

It is convenient to reexpress the remaining threedimensional phase space for (d)SPDC in terms of the signal emission angles $\theta_{s}$ and $\phi_{s}$ and the signal frequency (or, equivalently, $\alpha_{\omega}$ ). Within our assumptions, the distribution in $\phi_{s}$ is flat. With respect to the polar angle and 


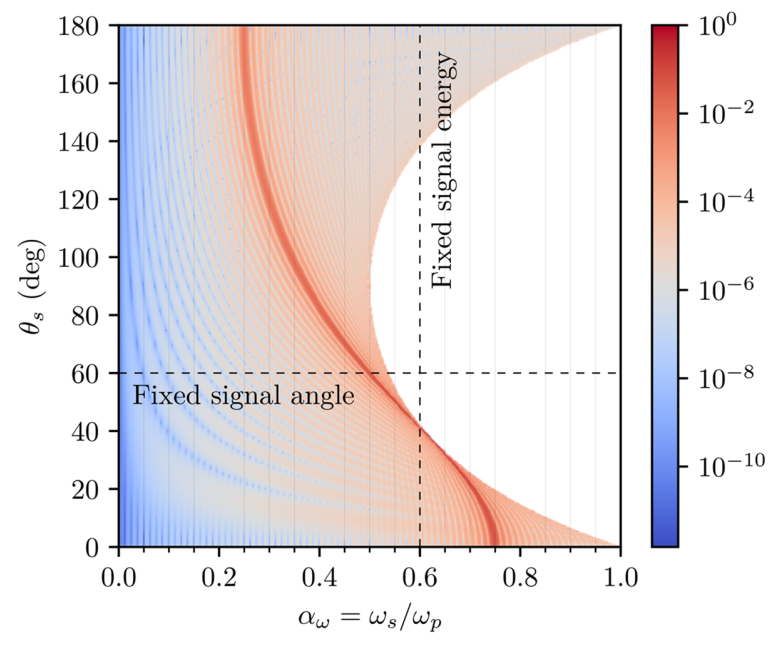

FIG. 4. The phase-space distribution $d^{2} \hat{\Gamma} / d\left(\cos \theta_{s}\right) d \alpha_{\omega}$ for SPDC in arbitrary units. The distribution is strongly peaked along the region of phase space in which phase matching is achieved. We use $L=1, \lambda_{p}=L / 10, n_{p}=2$ and $n_{s}=n_{i}=4$. These parameters are unrealistic in practice but allow for clear visualization of the distribution. The dashed lines show slices of fixed signal angle or energy.

frequency, we find that

$$
\begin{aligned}
& \frac{d^{2} \hat{\Gamma}}{d\left(\cos \theta_{s}\right) d \alpha_{\omega}} \\
& =\frac{2 \pi \omega_{p}^{3} \alpha_{\omega}^{2}\left(1-\alpha_{\omega}\right) n_{s}^{3} \tilde{n}_{i}^{2}}{\sqrt{\Xi^{2}-\alpha_{\omega}^{2} n_{s}^{2} \sin ^{2} \theta_{s}}} \sum_{ \pm} L^{2} \operatorname{sinc}^{2} \\
& \quad \times\left[\left(n_{p}-n_{s} \alpha_{\omega} \cos \theta_{s} \pm \sqrt{\Xi^{2}-n_{s}^{2} \alpha_{\omega}^{2} \sin ^{2} \theta_{s}}\right) \frac{\omega_{p} L}{2}\right],
\end{aligned}
$$

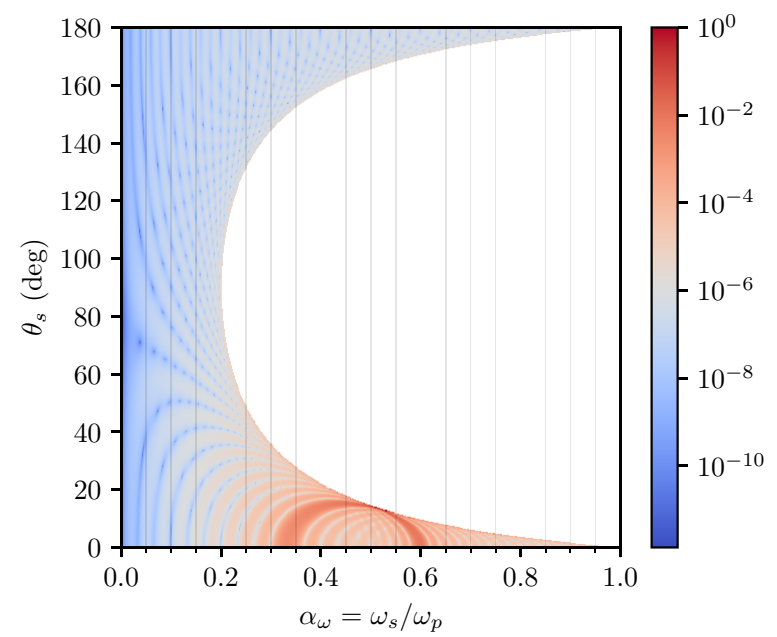

where

$$
\tilde{n}_{i} \stackrel{\text { def }}{=} \begin{cases}n_{i}, & \text { for SPDC, } \\ 1, & \text { for dSPDC }\end{cases}
$$

In Fig. 4, we show the double differential distribution $\hat{\Gamma}$ for SPDC and in Fig. 5 it is shown for dSPDC, both for a massless and a massive $\varphi$. As opposed to the infinitecrystal case, the violation of translation invariance by the finite extent of the crystal allows for a deviation from the exact phase-matching conditions. However, the distribution still clearly peaks in regions of phase space that satisfy the phase-matching conditions, those shown in Fig. 2. In addition to the sharp peak, an interference pattern is seen away for the phase-matching line when the momentum mismatch is a full or half-integer of the crystal length, captured by the sinc function in Eq. (27). To have a clearer view of these qualitative features in the distribution, we pick somewhat exaggerated values for indices of refraction in these figures.

\section{1. dSPDC searches with imaging or spectroscopy}

Though one can, in principle, measure both the angle and the energy of the signal photon, it is usually easier to measure either with a fixed angle or a fixed energy. For example, a CCD detector with a monochromatic filter can easily measure a single energy slice of this distribution, as shown in the vertical dashed line in Fig. 4. Likewise, a spectrometer with no spatial resolution can measure a fixed angle slice of this distribution, as shown in the horizontal dashed line of Fig. 4. An attractive choice for this is to look in the forward region, with an emission angle $\theta_{s}=0$, which is the case for waveguides. For this collinear case,

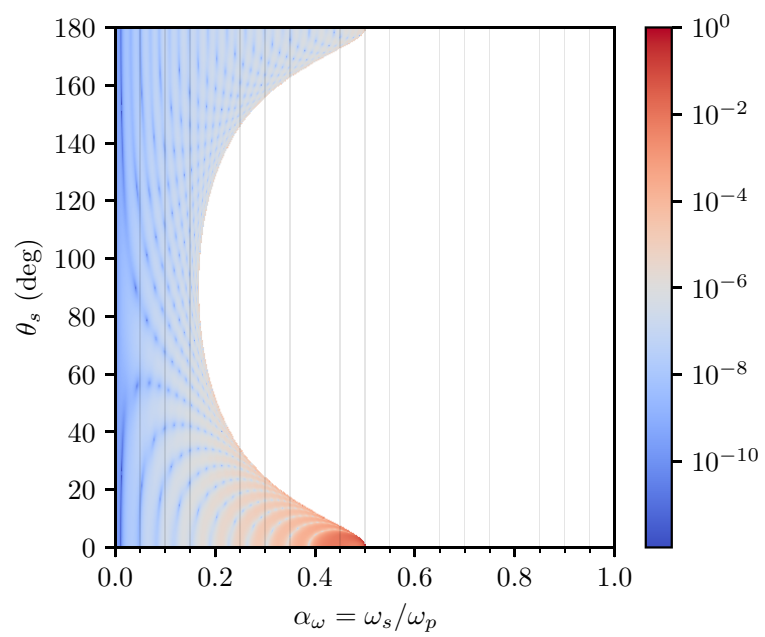

FIG. 5. The phase-space distribution $d^{2} \hat{\Gamma} / d\left(\cos \theta_{s}\right) d \alpha_{\omega}$ in arbitrary units for the dSPDC process for $m=0$ (left) and $m=0.5 \omega_{p}$ (right). The distributions are strongly peaked where the respective phase-matching conditions are satisfied. The other parameters are as in Fig. 4, with somewhat exaggerated indices of refraction to allow us to see the features in the distribution. 

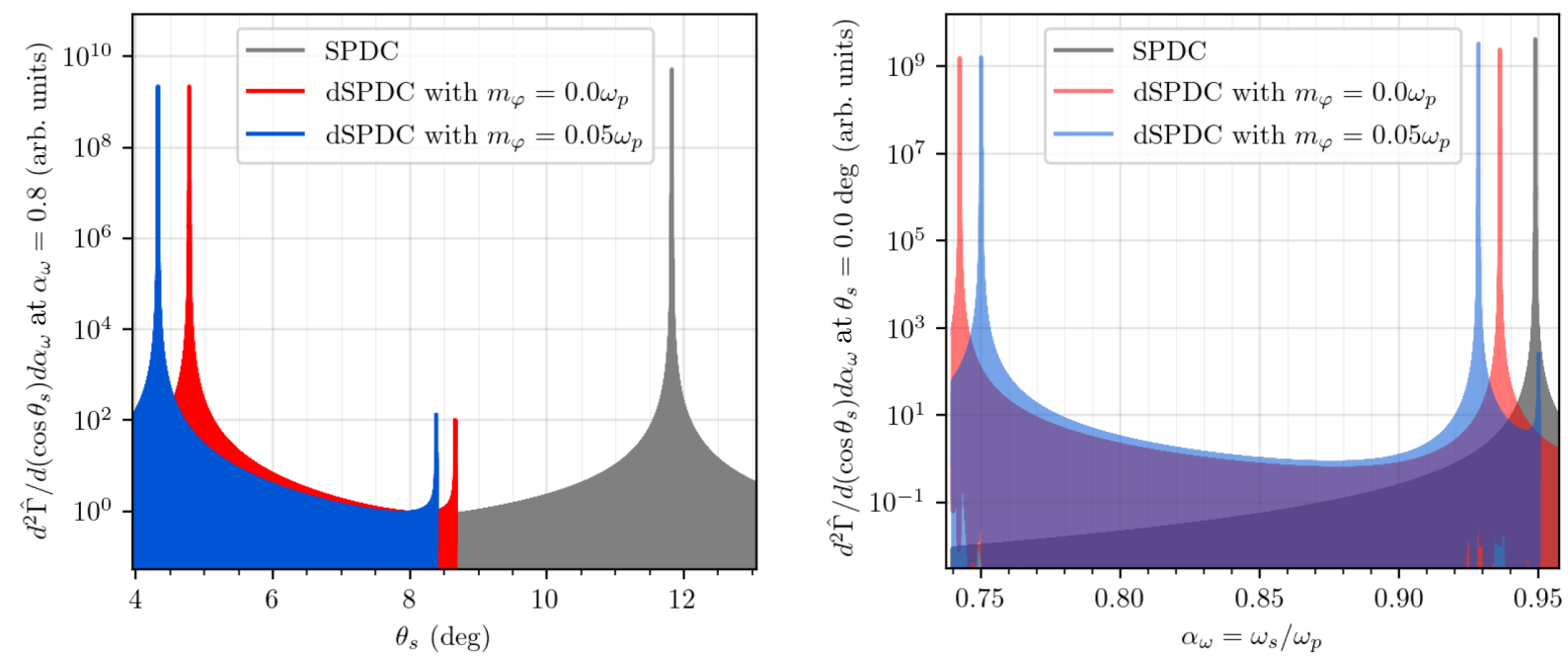

FIG. 6. Slices of the phase-space distributions for SPDC and for dSPDC with different choices of the $\varphi$ mass. On the left, we show angle distributions with fixed signal frequency, and on the right, we show frequency distributions for fixed signal angle. These distributions allow us to separate SPDC backgrounds from the dSPDC signal, as well as the dSPDC signal for different values of $m_{\varphi}$. The distributions become narrower for thicker crystals, enhancing the signal to background separation power. For these distributions, we use $L=1 \mathrm{~cm}, \lambda_{p}=400 \mathrm{~nm}$ and for the refractive indices $n_{p}=1.49$ and $n_{s}=n_{i}=1.66$, inspired by calcite.

we obtain a signal spectrum

$$
\frac{d \hat{\Gamma}}{d \alpha_{\omega}}=\frac{2 \pi \omega_{p}^{3} \alpha_{\omega}^{2}\left(1-\alpha_{\omega}\right) n_{s}^{3} \tilde{n}_{i}^{2}}{\Xi} \sum_{ \pm} L^{2} \operatorname{sinc}^{2}\left(\frac{\Delta k_{z \pm}^{(0)} L}{2}\right),
$$

where

$$
k_{z \pm}^{(0)}=k_{z p}-k_{z s}-k_{z i}^{ \pm}=\omega_{p}\left(n_{p}-n_{s} \alpha_{\omega} \pm \Xi\right)
$$

is the momentum mismatch for collinear (d)SPDC. In some cases, the collinear spectrum is dominated by just the forward emission of the idler or $\varphi$, while in others there are two phase-matching solutions that contribute similarly to the rate.

In Fig. 6, we show two distributions, one for fixed signal frequency and the other for a fixed signal angle, the latter in the forward direction. We see that both of these measurement schemes are able to distinguish the signal produced by different values of mass $m_{\varphi}$. Furthermore, in cases where the standard-model SPDC process is a source of background, it can be separated from the dSPDC signal. As expected, in the forward measurement the dSPDC spectrum exhibits two peaks for the emission of $\varphi$ in the forward or backward directions. It should be noted that the width of the highest peaks in these distributions decreases with crystal length $L$.

\section{OPTICAL MATERIALS FOR DARK SPDC}

Having discussed the phase-space distribution for dSPDC, we now discuss which optical media are needed to open this channel, to enhance its rates, and, if possible, to suppress the backgrounds. A more complete estimate of the dSPDC rate will be presented in Ref. [13]. Here, we discuss the various features qualitatively to enable optimization of dSPDC searches.

\section{A. Refractive indices}

As discussed above, the refractive index of the optical medium plays a crucial role. It opens the phase space for the dSPDC decay and determines the kinematics of the process.

In order to enhance the dSPDC rate, it is always desirable to have a setup in which the dSPDC phase-matching conditions that we discussed in Sec. III are accomplished. Since the left-hand side of Eq. (17) is the cosine of an angle, its right-hand side is restricted between \pm 1 , so that

$$
-1<\frac{n_{p}^{2}+\alpha_{\omega}^{2} n_{s}^{2}-\Xi^{2}}{2 \alpha_{\omega} n_{p} n_{s}}<1 .
$$

From this, we find that so long as $n_{s}>n_{p}$, there is a range of $\varphi$ mass values that can take part in dSPDC. As the difference $n_{s}-n_{p}$ is taken to be larger, a greater $\varphi$ mass can be produced and larger opening angles $\theta_{s}$ can be achieved.

Using the inequalities in Eq. (31), we can explore the range of desirable indices of refraction for a particular setup choice. For example, suppose that we use monochromatic filter for the signal at half the energy of the pump photons, $\alpha_{\omega}=\frac{1}{2}$. Figure 7 shows in blue the region where the phase-matching condition is satisfied for the SPDC process and in orange the region where the phase-matching condition is satisfied for dSPDC. As can be seen, dSPDC 


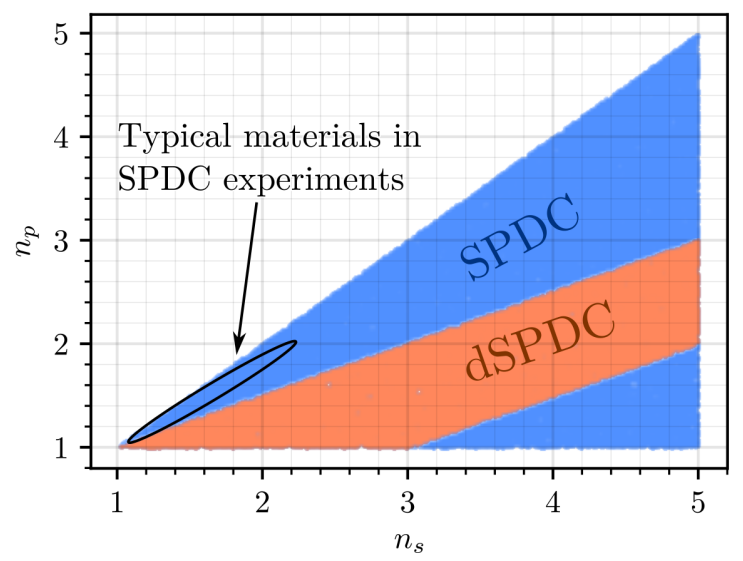

FIG. 7. The regions in which the refractive indices $n_{p}$ and $n_{s}$ allow the phase matching with $\alpha_{\omega} \equiv \omega_{s} / \omega_{p}=\frac{1}{2}$ for the SPDC process (in this case, assuming that $n_{i}=n_{s}$ ) and dSPDC (in this case, assuming that $\left.m /\left(\omega_{p}\right) \ll 1\right)$.

is more restrictive in the refractive indices. Furthermore, typical SPDC experiments employ materials such as beta barium borate (BBO), potassium dideuterium phosphate (KDP) and lithium triborate (LBO) [15,36-38] that do not allow the phase matching for dSPDC for this example.

A second example that is well motivated is the forward region, namely $\theta_{s}=0$. In this case, phase matching can be achieved so long as

$$
\frac{\left(n_{s}-n_{p}\right)^{2}}{n_{s}^{2}-1} \geq \frac{m_{\varphi}^{2}}{\omega_{p}^{2}}
$$

and $n_{s}>n_{p}$. We will investigate collinear dSPDC in greater detail in Ref. [13].

The dSPDC phase-matching requirement $n_{s}>n_{p}$ can be achieved in practice by several effects. The most common one, employed in the majority of SPDC experiments, is birefringence [36,38-40]. In this case, the polarization of each photon is used to obtain a different refractive index. For instance, in calcite, the ordinary and extraordinary polarizations have indices of refraction of $n_{o}=1.658$ and $n_{e}=1.486$. Taking the former to be the signal and taking the latter for the pump, phase matching can be met for $m_{\varphi}<0.16 \omega_{p}$. Birefringence may also be achieved in single-mode fibers and waveguides with noncircular cross section, e.g., polarization-maintaining optical fibers [41]. Of course, the dependence of the refractive index on the frequency may also be used to generate a signal-pump difference in $n$.

\section{B. Linear and birefringent media for axions}

Axion electrodynamics is itself a nonlinear theory. Therefore, dSPDC with emission of an axion can occur in a perfectly linear medium. The optical medium is needed in order to satisfy the phase-matching condition that cannot be satisfied in a vacuum. Because the pump and signal photons in axion dSPDC have different polarizations, a birefringent material can satisfy the requirement of $n_{s}>n_{p}$ and Eq. (32). Because SPDC can be an important background to a dSPDC axion search, the use of a linear or nearly linear medium, where SPDC is absent, is desirable. Interestingly, materials with a crystalline structure that is invariant under a mirror transformation $\mathbf{r} \rightarrow-\mathbf{r}$ will have a vanishing $\chi^{(2)}$ from symmetry considerations [42].

\section{Longitudinal susceptibility for dark-photon searches}

As opposed to the axion case, a dSPDC process with a dark photon requires a nonlinear optical medium. As a result, SPDC can be a background to dSPDC darkphoton searches. In SPDC, the pump, signal, and idler are standard-model photons and their polarization is restricted to be orthogonal to their propagation. As a consequence, when one wants to enhance an SPDC process, the nonlinear medium is oriented in such a way that the second-order susceptibility tensor $\chi^{(2)}$ can couple appropriately to the transverse polarization vectors of the pump, signal, and idler photons. The effective coupling between the modes in question is given by

$$
\chi_{\mathrm{SPDC}}^{(2)} \equiv \chi_{j k l}^{(2)} \varepsilon_{j}^{p} \varepsilon_{k}^{s} \varepsilon_{l}^{i},
$$

with the $\varepsilon$ being the (transverse) polarization vectors for the pump, signal, and idler photons. These are spanned by $(1,0,0)$ and $(0,1,0)$ in a frame in which the respective photon is propagating in the $\hat{z}$ direction.

In dSPDC with a dark photon, there is an important difference. Because the dark photon is massive, it can have a longitudinal polarization, $\varepsilon_{l}^{A_{L}^{\prime}}=(0,0,1)$. The crystal may be oriented to couple to this longitudinal mode, giving an effective coupling of

$$
\chi_{A_{L}^{\prime}}^{(2)} \equiv \chi_{j k l}^{(2)} \varepsilon_{j}^{p} \varepsilon_{k}^{s} \varepsilon_{l}^{A_{L}^{\prime}} .
$$

Maximization of this coupling benefits the search both by enhancing signal and reducing background. The background is reduced because the SPDC effective coupling to transverse modes given by Eq. (33) is suppressed. The signal is enhanced because the coupling to the longitudinal polarization of the dark photon is suppressed by $m_{A^{\prime}} / \omega$ rather than $\left(m_{A^{\prime}} / \omega\right)^{2}[32,33,43]$.

This motivates either nontraditional orientation of nonlinear crystals or the identification of nonlinear materials that would usually be ineffective for SPDC. As an example in the first category, any material that is used for typeII phase matching, in which the signal and idler have orthogonal polarization, can be rotated by $90^{\circ}$ to achieve a coupling of a longitudinal polarization to a pump and an idler. In the second category, material with $\chi^{(2)}$ tensors that 
are nonvanishing only in "maximally nondiagonal" $\hat{x}-\hat{y}-\hat{z}$ elements would obviously be discarded as standard SPDC sources but can have an enhanced dSPDC coupling.

\section{DARK-SPDC SETUPS AND RATES}

A precise estimate of the rate is beyond the scope of this work and will be discussed in more detail in Ref. [13]. Here, we rescale known rate formulas in order to examine the dependence of the rate on the geometric factors, such as the pump power and beam area, the crystal length, and the area and angle from which signal is collected.

Consider the geometry sketched in Fig. 8. A pump beam is incident on an optical element of length $L$ along the pump direction. The width of the pump beam is set by the laser parameters. The pump beam may consist of multiple modes or can be guided in a fiber or waveguide [12] and in a single mode. The width and angle of the signal "beam" is set by the apparatus used to collect and detect the signal. This too can include collection of a single mode in a fiber (see, e.g., Refs. [15,18]) or in multiple modes. In SPDC, when the idler photon may be collected, the width and angle of the idler can also be set by a similar apparatus. However, in dSPDC (or in SPDC if we choose to only collect the signal), the $\varphi$ beam is not a parameter in the problem. In this case, we are interested in an inclusive rate and would sum over a complete basis of idler beams. Such a sum will be performed in Ref. [13] but usually the sum will be dominated by a set of modes that are similar to those collected for the signal.

Generally, the signal rate will depend on all of the choices made above but we can make some qualitative observations. The rate for SPDC and ASPDC will be proportional to an integral over the volume defined by the overlap of the three beams in Fig. 8 and the crystal. When the length of the volume is set by the crystal, the process is said to occur in the "thin-crystal limit." In this limit, the beam overlap is roughly a constant over the crystal length

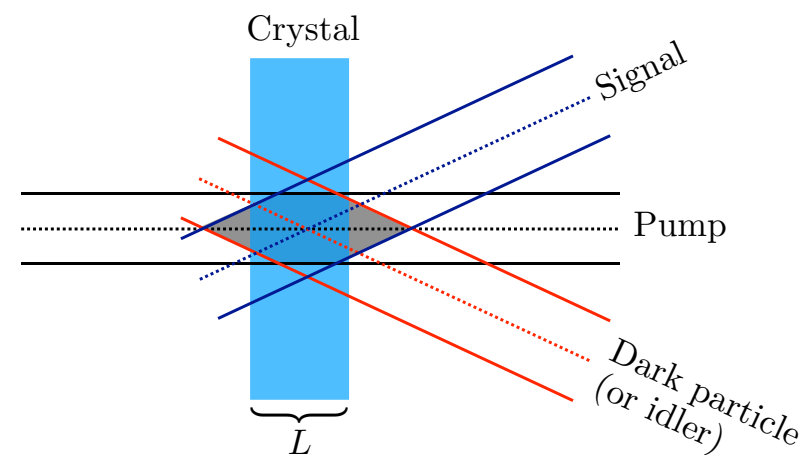

FIG. 8. A sketch showing the overlap of the pump, signal, and dark particle (or idler) beams with the crystal. In general, it is desirable to maximize the integration length, which is defined by the overlap of the three beams and the crystal. and thus the total rate will grow with $L$. Since dSPDC is a rare process, we observe that taking the collinear limit of the process, together with a thicker crystal, may allow for a larger integration volume and an enhanced rate. We note, however, that in order to benefit from the enhanced rate of the collinear process, one would need to contend with the bright pump beam, which spatially overlaps with the signal. Though this may be done with a combination of frequency and polarization selection, going to a (perhaps slightly) noncollinear configuration should also be considered.

The total rate for SPDC in a particular angle, integrated over frequencies, in the thin-crystal limit is $[13,15]$

$$
\Gamma_{\mathrm{SPDC}} \sim \frac{P_{p} \chi_{\mathrm{eff}}^{(2)^{2}} \omega_{s} \omega_{i} L}{\pi n_{p} n_{s} n_{i} A_{\mathrm{eff}}},
$$

where $L$ is the crystal length, $P_{p}$ is the pump power, $A_{\text {eff }}$ is the effective beam area, and $\chi_{\text {eff }}^{(2)}$ is the effective coupling of Eq. (33). A parametrically similar rate formula applies to SPDC in waveguides $[12,13]$. The pump power here is the effective power, which may be enhanced within a high-finesse optical cavity (see, e.g., Ref. [30]. The inverse dependence with effective area arrises because the interaction Hamiltonian is proportional to electric fields, which grow for fixed power for a tighter spot. For "bulk-crystal" setups, a pair production rate of the order of a few times $10^{6}$ per milliwatt of pump power per second is achievable [18] in the forward direction. In waveguided setups, in which the beams remain confined along a length of the order of a centimeter, production rates of order $10^{9}$ pairs per milliwatt per second are achievable in potassium titanyl phosphate (KTP) crystals [12,44], and rates of order $10^{10}$ have been discussed for lithium niobate [44]. Also noteworthy are cavity-enhanced setups, in which the effective pump power is increased by placing the device in an optical cavity, and/or OPOs, in which the signal photon is also a resonant mode, including compact devices with high conversion efficiencies (see, e.g., Refs. [45-48].

An important scaling of this rate is the $L / A_{\text {eff }}$ dependence. This scaling applies for the dSPDC rates discussed below. Within the thin-crystal limit, one can thus achieve higher rates with (a) a smaller beam spot and (b) a thicker crystal. It should be noted that for collinear SPDC, the crystal may be in the "thin limit" even for thick crystals (see Fig. 8 and imagine zero signal and idler emission angle). We now discuss the rates for dSPDC. We begin with the dark-photon case, which leads to higher rates at the chosen benchmark points.

\section{A. Dark-photon dSPDC rate}

The dSPDC rate into a dark photon with longitudinal polarization, $A_{L}^{\prime}$, can similarly be written as a simple 
rescaling of the above SPDC expression:

$$
\Gamma_{\mathrm{dSPDC}}^{\left(A_{L}^{\prime}\right)} \sim \epsilon^{2} \frac{m_{A^{\prime}}^{2}}{\omega_{A^{\prime}}^{2}} \frac{P_{p} \chi_{A_{L}^{\prime}}^{(2)^{2}} \omega_{s} \omega_{A^{\prime}} L}{n_{p} n_{S} A_{\mathrm{eff}}},
$$

where the effective coupling $\chi_{A_{L}^{\prime}}^{(2)}$ is defined in Eq. (34). This is valid in regions where the dark-photon mass is smaller than the pump frequency, such that the produced dark photons are relativistic and have a refractive index of 1 . Using the optimistic waveguide numbers above as a placeholder and assuming an optimized setup with similar $\chi^{(2)}$, the number of events expected after integrating over a time $t_{\text {int }}$ are

$$
N_{\text {events }}^{\left(A_{L}^{\prime}\right)} \sim 10^{21}\left(\epsilon^{2} \frac{m_{A^{\prime}}^{2}}{\omega_{A^{\prime}}^{2}}\right)\left(\frac{P_{p}}{\mathrm{~W}}\right)\left(\frac{L}{\mathrm{~m}}\right)\left(\frac{t_{\mathrm{int}}}{\text { year }}\right) .
$$

Benchmark points that saturate currant limits and corresponding examples of dSPDC waveguide setups are summarized in Table I. The current strongest laboratory-based limit for dark-photon masses of order $0.1 \mathrm{eV}$ is set by the ALPS experiment at $\epsilon \sim 3 \times 10^{-7}$. For this dark-photon mass $m_{A^{\prime}} \sim 0.1 \omega_{p}$, the $m_{A^{\prime}} / \omega_{A^{\prime}}$ term does not represent a large suppression. In this case, of the order of ten dSPDC events are produced in a day in a 1-cm crystal with $1 \mathrm{~W}$ of pump power with the above assumptions. This implies that a relatively small dSPDC experiment with an aggressive control on backgrounds could be used to push the current limits on dark photons.

Improvement of the limits from solar cooling, for which $\epsilon m_{A^{\prime}} / \omega_{A^{\prime}}$ is constrained to be smaller, would represent an interesting challenge. Achieving ten events in a year of running requires $1 \mathrm{~W}$ of power in a waveguide greater than $10 \mathrm{~m}$ (or a shorter waveguide with a higher stored power, perhaps using a Fabry-Perot setup). Interestingly, in terms of system size, this is still smaller than the ALPS II experiment, which would reach $100 \mathrm{~m}$ in length and an effecting power of a $100 \mathrm{~kW}$. This is because LSW setups require both production and detection, with limits scaling as $\epsilon^{4}$.

\section{B. Axion dSPDC rate}

A similar rate expression can be obtained for axion dSPDC:

$$
\Gamma_{\mathrm{dSPDC}}^{(\mathrm{axion})} \sim \frac{P_{p} g_{a \gamma \gamma}^{2} \omega_{s} L}{\omega_{\mathrm{axion}} n_{p} n_{s} A_{\mathrm{eff}}}
$$

where the different scaling with the frequency of the dark field is due to the different structure of the dSPDC interaction (recall that $\chi^{(2)}$ carries a mass dimension of -2 , while the dimension of the axion photon coupling is -1 ). Optimal SPDC (dSPDC) rates are achieved in waveguides in which the effective area is of the order of the square of the wavelength of the pump and signal light. Assuming a (linear) birefringent material that can achieve dSPDC phase matching for an axion, the number of signal event scales as

$$
N_{\text {events }}^{\text {(axion) }} \sim 40\left(\frac{g_{a \gamma}}{10^{-6} \mathrm{GeV}^{-1}}\right)^{2}\left(\frac{P_{p}}{\mathrm{~W}}\right)\left(\frac{L}{\mathrm{~m}}\right)\left(\frac{t_{\mathrm{int}}}{\text { year }}\right) .
$$

Benchmark points and example setups for axion dSPDC are summarized in Table I. This rate suggests that a dSPDC setup is promising in setting new laboratory-based limits on ALPs. For example, a 10-m birefringent single-mode fiber with $1 \mathrm{~kW}$ of pump power will generate of the order of ten events per day for couplings of order $10^{-7} \mathrm{GeV}^{-1}$. To probe beyond the CAST limits in $g_{a \gamma}$ may be possible and requires a larger setup, but not exceeding the scale, say, of ALPS II. In a 100-m length and an effective pump power of $100 \mathrm{~kW}$, a few dSPDC signal events are expected in a year. Maintenance of a low background would, of course, be crucial. We note, however, that optical fibers are routinely used over much greater distances, maintaining coherence (see, e.g., Ref. [5]), and an optimal setup should be identified.

\section{Backgrounds to dSPDC}

There are several factors that should be considered for the purpose of reducing backgrounds to SPDC:

(a) The crystal length and signal bandwidth. In addition to the growth of the signal rate, the signal bandwidth in many setups will decrease with $L$. If this is achieved, the signal-to-background ratio in a narrow band around the dSPDC phase-matching solutions will scale as $L^{2}$.

(b) The timing. The dSPDC signal consists of a single photon, whereas SPDC backgrounds will consist of two coincident photons. Backgrounds can be reduced using fast detectors and by rejecting coincidence events.

(c) The optical material. As pointed out in Sec. IV, purely linear birefringent materials can be used to reduce SPDC backgrounds to axion searches. Nonlinear materials with a $\chi^{(2)}$ tensor that couples purely to longitudinal polarizations may be used to enhance dark-photon dSPDC events without enhancing SPDC. This technique to reduce SPDC may also be used in axion searches.

(d) Higher-order nonlinearities. Though reflection symmetry may be used to reduce three-photon interactions, higher-order nonlinearities, such as fourwave mixing, will likely be present at some level and may contribute to backgrounds. For example, a four-photon interaction may contribute to a one- to three-photon transition, which would enable deviations from the one-to-two phase-matching relations 
TABLE I. The current laboratory-based and solar-based limits on the couplings of dark photons and axionlike particles with a benchmark mass of $0.1 \mathrm{eV}$. For each limit, we show the parameters of an example dSPDC in a waveguide and the rate it would produce for couplings that would produce the specified benchmark rate with the corresponding coupling. The pump power is an effective power, which can include an enhancement by an optical cavity setup. For dark-photon rates, we assume a nonlinearity of the same order as found in KTP crystals.

\begin{tabular}{lcc}
\hline \hline Current limits & $\begin{array}{c}\text { Dark photon } \\
\left(m_{A^{\prime}}=0.1 \mathrm{eV}\right)\end{array}$ & $\begin{array}{c}\text { Axionlike particle } \\
\left(m_{a}=0.1 \mathrm{eV}\right)\end{array}$ \\
\hline Laboratory & $\epsilon<3 \times 10^{-7}$ & $g_{a \gamma}<10^{-6} \mathrm{GeV}^{-1}$ \\
Example dSPDC setup & $P_{p}=1 \mathrm{~W}$ & $P_{p}=1 \mathrm{~kW}$ \\
& $L=1 \mathrm{~cm}$ & $L=10 \mathrm{~m}$ \\
& $\Gamma=10$ per day & $\Gamma=10$ per day \\
\hline Solar & $\epsilon<10^{-10}$ & $g_{a \gamma}<10^{-10} \mathrm{GeV}^{-1}$ \\
Example dSPDC setup & $P_{p}=1 \mathrm{~W}$ & $P_{p}=100 \mathrm{~kW}$ \\
& $L=10 \mathrm{~m}$ & $L=100 \mathrm{~m}$ \\
& $\Gamma=10$ per year & $\Gamma=10$ per year \\
\hline \hline
\end{tabular}

due to the presence of an additional photon in the final state. Such a conversion process would, however, lead to a continuum spectrum of signal photons, as opposed to the sharp lines, say, of Fig. 6. We further note that spectral features can arise due to four-wave-mixing at frequencies - say, with zero dispersion (see, e.g., Ref. [49]) — and such effects would need to be characterized and understood.

(e) Detector noise and optical impurities. Sources of background that may be a limiting factor for dSPDC searches include detector noise, as well as scattering of pump photons off impurities in the optical elements and surfaces.

An optimal dSPDC-based search for dark particles will likely consider these factors and is left for future investigation.

\section{DISCUSSION AND CONCLUSIONS}

We present a method to search for new light and feebly coupled particles, such as axionlike particles and dark photons. The dSPDC process allows us to tag the production of a dark state as a pump photon down-converts to a signal photon and a "dark idler" $\varphi$, in close analogy to SPDC. We show that the presence of indices of refraction that differ from one open the phase space for the decay, or down-conversion, of the massless photon to the signal plus the dark particle, even if $\varphi$ has a mass. This type of search has a parametric advantage over LSW setups, since it only requires the production of the axion or dark photon, without the need to detect it again. In addition, axion searches based on ASPDC do not require an external background magnetic field. Precise sensitivity calculations for dSPDC with dark-photon and axion cases that can be achieved through this method are ongoing [13].
The commonplace use of optics in telecommunications, imaging, and quantum information science, as well as the development of advanced detectors, can thus be harnessed to search for dark-sector particles. Our dSPDC proposal is a first step to bring these areas into the fold of dark-particle searches. Increasing dSPDC signal rates will require high laser power and long optical elements. Enhancing the signal and suppressing SPDC backgrounds also requires identification of the right optical media for the search. Axion searches would benefit from optically linear and birefringent materials, with greater birefringence allowing us to search for higher axion masses. Searches for dark photons would benefit from strongly nonlinear materials that are capable of coupling to a longitudinal polarization. This, in turn, motivates either nonconventional optical media or the use of conventional crystals that are oriented by a $90^{\circ}$ rotation from that which is desired for SPDC. Enhancement of the effective pump power with an optical cavity is a straightforward way to enhance the dSPDC rate. We leave the exploration of a "doubly resonant" OPO setup in which the signal is also a cavity eigenmode (in parallel with Ref. [30]) for future work. In addition, the exploitation of four-wave mixing for dark-particle searches warrants further exploration. Finally, the detection of rare signal events requires sensitive single-photon detectors with high spatial and/or frequency resolution.

Given the substantial effort in searching for axions and dark photons, new and complementary approaches to the search, which harness new areas of technology, are desirable. The dSPDC process possesses many experimental handles by which a potential signal can be cross checked. The signal is expected to vary in specific ways by changing the crystal length, the beam parameters, the indices of refraction, and the pump frequency. The spectral and/or angular dependence of the signal also exhibits sharp features. Of course, even in the absence of a positive signal, a new dSPDC-based limit on dark photons or axions is an important component in the search for new light particles interacting with photons. We conclude that phase-space engineering using quantum and nonlinear optics tools is a promising new technique for future explorations of dark-sector parameter spaces.

\section{ACKNOWLEDGMENTS}

We thank Joe Chapman, Paul Kwiat, and Neal Sinclair for informative discussions. This work was funded by a Department of Energy (DOE) Quantum Information Science Enabled Discovery (QuantISED) grant.

[1] A. Furusawa, Unconditional quantum teleportation, Science 282, 706 (1998).

[2] D. Boschi, S. Branca, F. De Martini, L. Hardy, and S. Popescu, Experimental Realization of Teleporting an 
Unknown Pure Quantum State via Dual Classical and Einstein-Podolsky-Rosen Channels, Phys. Rev. Lett. 80, 1121 (1998).

[3] D. Bouwmeester, J.-W. Pan, K. Mattle, M. Eibl, H. Weinfurter, and A. Zeilinger, Experimental quantum teleportation, Nature 390, 575 (1997).

[4] C. H. Bennett, G. Brassard, C. Crépeau, R. Jozsa, A. Peres, and W. K. Wootters, Teleporting an Unknown Quantum State via Dual Classical and Einstein-Podolsky-Rosen Channels, Phys. Rev. Lett. 70, 1895 (1993).

[5] R. Valivarthi et al., Teleportation Systems Towards a Quantum Internet. July 2020 arXiv e-prints, page arXiv:2007.11157.

[6] A. G. White, J. R. Mitchell, O. Nairz, and P. G. Kwiat, Interaction-free imaging, Phys. Rev. A 58, 605 (1998).

[7] C. K. Hong and L. Mandel, Theory of parametric frequency down conversion of light, Phys. Rev. A 31, 2409 (1985).

[8] J. A. Giordmaine and R. C. Miller, Tunable Coherent Parametric Oscillation in $\mathrm{LiNbO}_{3}$ at Optical Frequencies, Phys. Rev. Lett. 14, 973 (1965).

[9] R. Essig, J. A. Jaros, W. Wester, P. H. Adrian, S. Andreas, T. Averett, O. Baker, B. Batell, M. Battaglieri, J. Beacham, and T. Beranek, Dark Sectors and New, Light, WeaklyCoupled Particles. arXiv:1311.0029 [astro-ph, physics:hepex, physics:hep-ph], October 2013. arXiv:1311.0029.

[10] F. Hoogeveen and T. Ziegenhagen, Production and detection of light bosons using optical resonators, Nucl. Phys. B 358, 3 (1991).

[11] Searches for missing energy and momentum are a commonplace tool in the search for new physics at colliders [50-54]. However, the dSPDC kinematics are distinct, as we show.

[12] M. Fiorentino, S. M. Spillane, R. G. Beausoleil, T. D. Roberts, P. Battle, and M. W. Munro, Spontaneous parametric down-conversion in periodically poled KTP waveguides and bulk crystals, Opt. Express 15, 7479 (2007).

[13] J. Estrada, R. Harnik, D. Rodrigues, and M. Senger, New Particle Searches with Nonlinear Optics. In preparation.

[14] L. Phuong Hoang and X. Binh Cao, Signal detection using biphotons and potential application in axion-like particle search, Nucl. Phys. B 961, 115215 (2020).

[15] A. Ling, A. Lamas-Linares, and C. Kurtsiefer, Absolute emission rates of spontaneous parametric down conversion into single transverse Gaussian modes, Phys. Rev. A 77, 043834 (2008). ArXiv:0801.2220.

[16] J. Schneeloch and J. C. Howell, Introduction to the transverse spatial correlations in spontaneous parametric downconversion through the biphoton birth zone, J. Opt. 18, 053501 (2016).

[17] P. Norman and K. Ruud, Microscopic theory of nonlinear optics. Non-Linear Optical Properties of Matter, p. 1-49, 2006.

[18] R. S. Bennink, Optimal co-linear Gaussian beams for spontaneous parametric down-conversion, Phys. Rev. A 81, 053805 (2010). ArXiv:1003.3810.

[19] R. D. Peccei and H. R. Quinn, CP Conservation in the Presence of Pseudoparticles, Phys. Rev. Lett. 38, 1440 (1977).

[20] P. Svrcek and E. Witten, Axions in string theory, JHEP 06, 051 (2006).
[21] P. W. Graham, I. G. Irastorza, S. K. Lamoreaux, A. Lindner, and K. A. van Bibber, Experimental searches for the axion and axion-like particles, Ann. Rev. Nucl. Part. Sci. 65, 485 (2015).

[22] B. Holdom, Two U(1)'s and epsilon charge shifts, Phys. Lett. B 166, 196 (1986).

[23] P. A. Zyla, R. M. Barnett, J. Beringer, O. Dahl, D. A. Dwyer, D. E. Groom, C. J. Lin, K. S. Lugovsky, E. Pianori, and D. J. Robinson, Review of particle physics, PTEP 2020, 083C01 (2020).

[24] G. G. Raffelt, Astrophysical methods to constrain axions and other novel particle phenomena, Phys. Rept. 198, 1 (1990).

[25] V. Anastassopoulos, S. Aune, K. Barth, A. Belov, G. Cantatore, J. M. Carmona, J. F. Castel, S. A. Cetin, F. Christensen, J. I. Collar, and T. Dafni, New CAST limit on the axion-photon interaction, Nat. Phys. 13, 584 (2017).

[26] A. Abeln, N. Ushakov, C. J. Hailey, J. P. Mols, V. Muratova, J. F. Castel, U. Werthenbach, N. Bykovskiy, J. MiraldaEscudé, O. Limousin, and I. Fleck, Conceptual Design of BabyIAXO, the intermediate stage towards the International Axion Observatory. 102020.

[27] E. Armengaud, F. T. Avignone, M. Betz, P. Brax, P. Brun, G. Cantatore, J. M. Carmona, G. P. Carosi, F. Caspers, S. Caspi, and S. A. Cetin, Conceptual design of the International Axion Observatory (IAXO), JINST 9, T05002 (2014).

[28] F. Della Valle, A. Ejlli, U. Gastaldi, G. Messineo, E. Milotti, R. Pengo, G. Ruoso, and G. Zavattini, The PVLAS experiment: Measuring vacuum magnetic birefringence and dichroism with a birefringent Fabry-Perot cavity, Eur. Phys. J. C 76, 24 (2016).

[29] R. Ballou, G. Deferne, M. Finger Jr, M. Finger, L. Flekova, J. Hosek, S. Kunc, K. Macuchova, K. A. Meissner, P. Pugnat, and M. Schott, New exclusion limits on scalar and pseudoscalar axionlike particles from light shining through a wall, Phys. Rev. D 92, 092002 (2015).

[30] K. Ehret, et al., Resonant laser power build-up in ALPS: A "light-shining-through-walls" experiment, Nucl. Instrum. Meth. A 612, 83 (2009).

[31] R. Bähre, et al., Any light particle search II-technical design report, JINST 8, T09001 (2013).

[32] H. A. Maxim Pospelov and J. Pradler, New stellar constraints on dark photons, Phys. Lett. B 725, 190 (2013).

[33] H. A. Maxim Pospelov and J. Pradler, Dark Matter Detectors as Dark Photon Helioscopes, Phys. Rev. Lett. 111, 041302 (2013).

[34] D. Tong, Quantum Field Theory. http://www.damtp.cam. ac.uk/user/tong/qft.html.

[35] The meaning of what constitutes the thin-crystal-layer limit in our context is discussed in Sec. V.

[36] P. G. Kwiat, E. Waks, A. G. White, I. Appelbaum, and P. H. Eberhard, Ultra-bright source of polarizationentangled photons, Phys. Rev. A 60, R773 (1999). ArXiv:quant-ph/9810003.

[37] C. Couteau, Spontaneous parametric down-conversion, Contemp. Phys. 59, 291 (2018).

[38] N. Boeuf, Calculating characteristics of noncollinear phase matching in uniaxial and biaxial crystals, Opt. Eng. 39, 1016 (2000). 
[39] P.-A. Moreau, E. Toninelli, T. Gregory, and M. J. Padgett, Imaging with quantum states of light, Nat. Rev. Phys. 1, 367 (2019).

[40] S. Magnitskiy, D. Frolovtsev, V. Firsov, P. Gostev, I. Protsenko, and M. Saygin, A SPDC-based source of entangled photons and its characterization, J. Russ. Laser Res. 36, 618 (2015).

[41] J. Noda, K. Okamoto, and Y. Sasaki, Polarizationmaintaining fibers and their applications, J. Lightwave Technol. 4, 1071 (1986).

[42] R. W. Boyd, Nonlinear Optics (Elsevier, Amsterdam, 2008), 3rd ed.

[43] P. W. Graham, J. Mardon, S. Rajendran, and Y. Zhao, Parametrically enhanced hidden photon search, Phys. Rev. D 90, 075017 (2014).

[44] S. M. Spillane, M. Fiorentino, and R. G. Beausoleil, Spontaneous parametric down conversion in a nanophotonic waveguide, Opt. Express 15, 8770 (2007).

[45] J. U. Fürst, D. V. Strekalov, D. Elser, A. Aiello, U. L. Andersen, Ch. Marquardt, and G. Leuchs, Low-Threshold Optical Parametric Oscillations in a Whispering Gallery Mode Resonator, Phys. Rev. Lett. 105, 263904 (2010).

[46] T. Beckmann, H. Linnenbank, H. Steigerwald, B. Sturman, D. Haertle, K. Buse, and I. Breunig, Highly Tunable Low-Threshold Optical Parametric Oscillation in Radially Poled Whispering Gallery Resonators, Phys. Rev. Lett. 106, 143903 (2011).
[47] C. S. Werner, K. Buse, and I. Breunig, Continuous-wave whispering-gallery optical parametric oscillator for highresolution spectroscopy, Opt. Lett. 40, 772 (2015).

[48] J. L. Ayed Al Sayem, Z. Gong, J. B. Surya, C.-L. Zou, and H. X. Tang, Ultralow-threshold thin-film lithium niobate optical parametric oscillator, 2021.

[49] K. Inoue, Four-wave mixing in an optical fiber in the zerodispersion wavelength region, J. Lightwave Technol. 10, 1553 (1992).

[50] J. Goodman, M. Ibe, A. Rajaraman, W. Shepherd, T. M. P. Tait, and H.-B. Yu, Constraints on light Majorana dark matter from colliders, Phys. Lett. B 695, 185 (2011).

[51] Y. Bai, P. J. Fox, and R. Harnik, The Tevatron at the frontier of dark matter direct detection, JHEP 12, 048 (2010).

[52] J. Goodman, M. Ibe, A. Rajaraman, W. Shepherd, T. M. P. Tait, and H.-B. Yu, Constraints on dark matter from colliders, Phys. Rev. D 82, 116010 (2010).

[53] P. J. Fox, R. Harnik, J. Kopp, and Y. Tsai, Missing energy signatures of dark matter at the LHC, Phys. Rev. D 85, 056011 (2012).

[54] T. Aaltonen, B. Á. González, S. Amerio, D. Amidei, A. Anastassov, A. Annovi, J. Antos, G. Apollinari, J. A. Appel, T. Arisawa, and A. Artikov, A Search for Dark Matter in Events with One Jet and Missing Transverse Energy in $p \bar{p}$ Collisions at $\sqrt{s}=1.96 \mathrm{TeV}$, Phys. Rev. Lett. 108, 211804 (2012). 